\title{
A UTILIZAÇÃO DO DELINEAMENTO DE LINHA DE BASE MÚLTIPLA PARA AVALIAÇÃO DAS HABILIDADES MANIPULATIVAS DO BADMINTON EM UM ALUNO COM SÍNDROME DE DOWN.
}

Amália Rebouças de Paiva e Oliveira, Adriana Garcia Gonçalvez, Manoel Osmar Seabra Junior.

Universidade Federal de São Carlos - UFSCAR, Mestrado em Educação, SP. E-mail: amaliareboucas@gmail.com.

\section{RESUMO}

O presente trabalho buscou analisar como o delineamento do sujeito único de linha de bases múltiplas pode se instrumento de avaliação na aquisição das habilidades manipulativas do badminton: forehand; backhand; e saque. Para tanto foi aplicado um protocolo de observação no qual foram registrados os erros e acertos em cinco tentativas de execução da tarefa para cada habilidade. Por meio desse protocolo foram estabelecidos a aprendizagem durante a linha de base, as intervenções e a manutenção do comportamento. Observou-se que o delineamento em questão foi eficaz para acompanhar a aprendizagem do adolescente com síndrome de Down nas habilidades manipulativas do badminton.

Palavras chave: delineamento do sujeito único; habilidades manipulativas; badminton; síndrome de Down.

THE USE OF MULTIPLE-BASED LINE DESIGN FOR ASSESSING BADMINTON MANIPULATIVE SKILLS IN A STUDENT WITH DOWN SYNDROME.

\begin{abstract}
The present work sought to analyze how the delineation of the single subject of multiple base line can be an instrument of evaluation in the acquisition of the manipulative skills of badminton: forehand; backhand; And serve. In order to do so, an observation protocol was applied in which the errors and correctness were registered in five attempts to execute the task for each skill. Through this protocol, learning during the baseline, interventions and behavior maintenance were established. It was observed that the design in question was effective to accompany the learning of the adolescent with Down syndrome in the manipulative skills of badminton.
\end{abstract}

Keywords: single subject delineation; Manipulative skills; badminton; Down's syndrome. 


\section{INTRODUÇÃO}

A síndrome de Down é caracterizada como uma alteração genética na qual resulta em um cromossomo a mais no par 21 que resulta em determinadas características. (DALLA DÉA; BALDIN;DALLA DÉA, 2009 ; SCHWARTZMAN, 1999). Entre as várias características próprias da síndrome, é possível destacar a presença de alteração do tônus muscular (hipotonia) e um déficit no desenvolvimento motor (ORNELAS; SOUSA, 2001). Tais características estão intrinsicamente ligadas. Uma alternativa que visa oferecer estímulos para potencializar o desenvolvimento motor da pessoa com síndrome de Down é a atividade física e o esporte. Vários autores apontam o esporte como fator determinante para o desenvolvimento global de pessoas com síndrome de Down (FLORES; BANKOFF 2010) (SILVA; FERREIRA 2001) (REIS FILHO E SCHULLER 2010) (LEITÃO 1999) (LIMA 2010) (PINTO 2013) (APOLONI ; LIMA; VIERA 2013).

Entretanto, ao trabalhar o esporte com pessoas com síndrome de Down, é importante desenvolver ferramentas e estratégias para avaliar seu desenvolvimento. Assim, o professor pode adequar o programa de ensino as reais necessidades do aluno. Neste panorama, surge a seguinte problemática: como avaliar a aprendizagem de uma tarefa motora durante um programa de ensino, mais especificamente, a tarefa motora de rebater no badminton.

O presente estudo, abordou a modalidade do badminton, caracterizado como um esporte de raquete que objetiva acertar a quadra adversária com a peteca, marcando assim sua pontuação (DUARTE, 2004). O badminton é um esporte capaz de oferecer uma gama de estímulos que auxiliem no desenvolvimento de pessoas com e sem deficiência. Na tarefa motora de rebater a peteca no badminton, destaca-se três movimentos: forehand; backhand; e saque; que serão explorados na metodologia.

\section{OBJETIVO}

Este estudo transversal, realizado a partir da dissertação de mestrado intitulada "Programa de iniciação ao badminton em adolescentes com síndrome de Down" (OIVEIRA,2015), buscou analisar a eficácia do delineamento do sujeito único na avaliação da aquisição da tarefa motora de rebater na modalidade do badminton quando ensinada a um adolescente com síndrome de Down.

\section{METODOLOGIA}

\section{Tipologia do Estudo}

Para a presente pesquisa foi utilizado o delineamento do sujeito único, mais especificamente o delineamento de linha de base múltipla entre comportamentos (GAST, 2010), no qual o indivíduo e as condições são as mesmas, porém, são analisadas diferentes habilidades. Nesse tipo de delineamento os sujeitos são expostos a condições que são mensuradas repetidamente. A prática do badminton envolve inúmeras habilidades. Para o presente estudo foram escolhidas as habilidades manipulativas do badminton: forehand; backhand; e saque.

Matos (1999 p.15), detalha a coleta de dados nesse tipo de delineamento:

Uma linha de base é estabelecida para cada um de diferentes comportamentos, e uma intervenção é planejada para cada um destes comportamentos, mas esta intervenção é introduzida em diferentes momentos (MATOS, 1999, p.15).

Dessa maneira, enquanto a intervenção é realizada com uma habilidade, as outras habilidades permanecem em linha de base e/ou manutenção. Para Cozby (2003) a linha de base compreende a mensuração do comportamento anterior ao período de manipulação das variáveis, ou seja, a linha de base é estabelecida para que a pesquisadora consiga avaliar o desenvolvimento dos alunos ao introduzir o programa de ensino. Após a manipulação das variáveis, ocorre o 
período de manutenção, onde são realizadas algumas medidas para verificar se o aluno continua executando a habilidade ou o comportamento analisado mesmo com o término das intervenções.

3.2 Procedimentos para coleta de dados

A presente pesquisa foi submetida ao Comitê de Ética em Pesquisas com Seres Humanos da Universidade Federal de São Carlos por meio da Plataforma Brasil, conforme a Resolução no 196/96 e resolução no 466, de 12 de dezembro de 2012 do Conselho Nacional de Saúde e obteve aprovação com o parecer n.769.763.

Para análise da tarefa motora de rebater no badminton, foram analisadas três habilidades: forehand; backhand; e saque, como ilustra a figura a seguir:

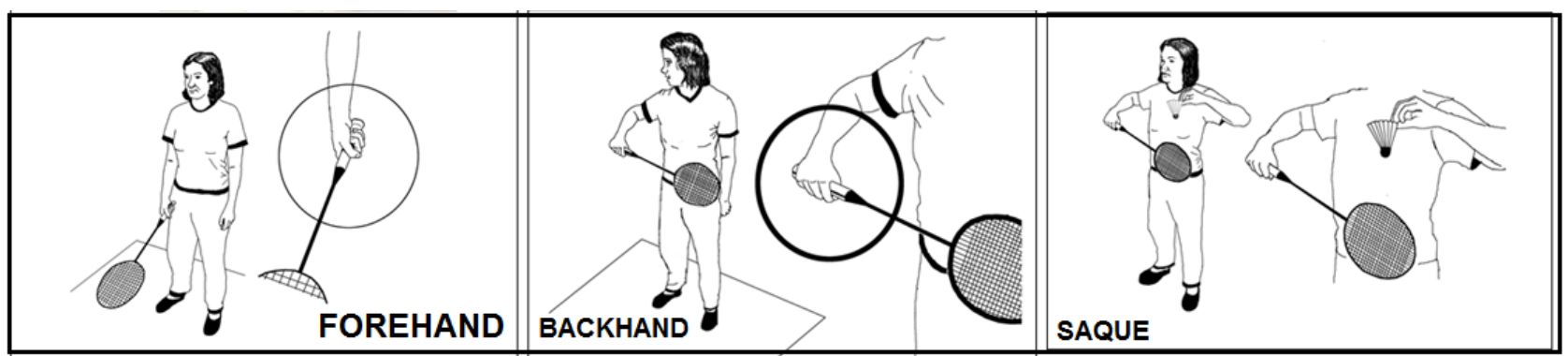

Figura 1. Ilustração do forehand; backhand; e saque Fonte: elaboração própria

Para linha de base e posterior avaliação a peteca foi lançada pela pesquisadora a 1,5 metros de distância do aluno no forehand e backhand, e para as medidas do saque, foi requisitado verbalmente ao aluno que executasse o movimento do saque do badminton. As três habilidades trabalhadas passaram pela seguinte sequência: linha de base $\rightarrow$ intervenção $\rightarrow$ manutenção.

Foi elaborado um protocolo de observação para o registro dos erros e acertos dos alunos. Este instrumento foi utilizado durante toda a pesquisa, tanto para a coleta da linha de base, bem como, para registro de acertos e erros após cada intervenção. O protocolo aborda as três habilidades que foram trabalhadas: forehand; backhand; e saque. Em todas as habilidades o aluno teve cinco oportunidades para realizar a tarefa, bem como a pesquisadora ofereceu o comando verbal para cada atividade pré-estipulada no protocolo de observação e forneceu ao aluno a demonstração do movimento. Todas as sessões, bem como os testes realizados pelo protocolo de observação, foram registradas em vídeo. Para avançar o ensino da habilidade foi estipulado como critério atingir $60 \%$ de acertos em pelo menos duas sessões.

O programa de iniciação ao badminton teve duração de oito semanas ${ }^{1}$, com duas intervenções semanais de 30 minutos de duração. Para habilidade do forehand foram necessárias quatro sessões de ensino, para o backhand foram necessárias cinco sessões de ensino, para o saque foram realizadas quatro sessões. Cada sessão de ensino foi composta de três atividades. As atividades compreendiam realizar a rebatida do forehand e do backhand, e realizar o saque com diferentes recursos pedagógicos. Todas as atividades previstas começavam por um baixo índice de complexidade e aumentava progressivamente.

\section{RESULTADOS E DISCUSSÃO}

A aplicação do protocolo de observação no aluno gerou o gráfico a seguir. No eixo Y está a porcentagem de acerto e erro que varia entre $0 \%$ a $100 \%$. No eixo X está a quantidade de sessões realizadas com o aluno em cada fase do programa de ensino. Ressalta-se que em todos os encontros foram aplicados o protocolo de observação para coleta de acertos e erros.

\footnotetext{
${ }^{1} \mathrm{O}$ programa de ensino completo encontra-se descrito na dissertação. Para acessar segue o link:

https://repositorio.ufscar.br/bitstream/handle/ufscar/7768/DissARPO.pdf?sequence=1\&isAllowed=y
} 


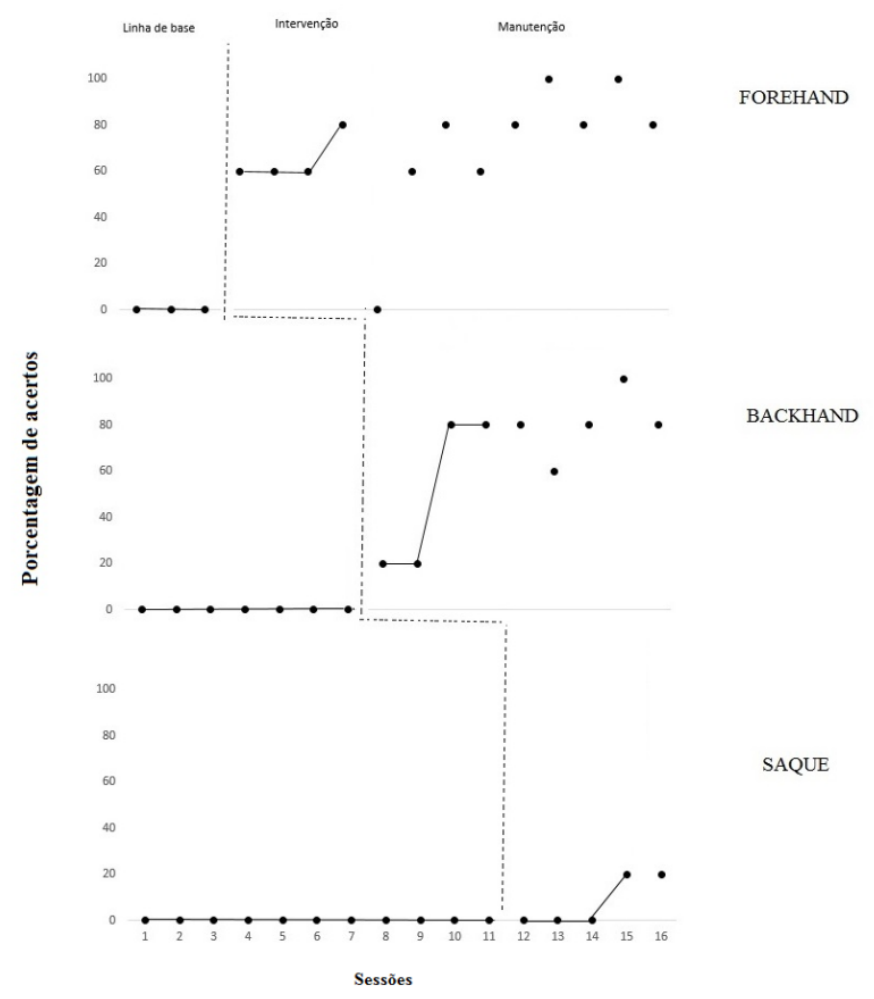

Gráfico 1. Distribuição de Frequência absoluta e relativa segundo o número de sessões e os acertos e erros do aluno em cada sessão, respectivamente.

Fonte: elaboração própria.

Observe que na linha de base da habilidade do forehand, o aluno A permaneceu na pontuação de $0 \%$ de acertos. Foram realizadas quatro sessões de ensino dessa habilidade. Também foram realizadas quatro sessões de ensino do backhand e quatro do saque, e em ambas as habilidades a linha de base permaneceu estável. É possível observar que o aluno A avançou de pontuação no forehand, e no backhand com a introdução da intervenção

$O$ aluno apresentou grande dificuldade na habilidade do saque, e não conseguiu atingir a meta proposta de $60 \%$ de acertos. A habilidade do saque é considerada uma habilidade mais complexa, pois depende da combinação de dois movimentos distintos dos membros superiores: o lançamento da peteca e a rebatida da mesma. Gallahue e Ozmum (2005) salientam que no estágio de combinação do aprendizado de uma nova habilidade motora o aluno deve "integrar habilidades múltiplas numa sequência espaço/tempo" (GALAHHUE; OZMUM, p.382). O aluno compreendia que se tratava da combinação de dois movimentos, entretanto não conseguia realizar a execução. O aluno colocava a peteca em cima da raquete, e posteriormente levantava a raquete arremessando assim a peteca.

As habilidades motoras dos esportes de raquete são definidas para Gallahue e Ozmun (2005) como habilidades motoras especializadas. Para os autores "o desenvolvimento de habilidades motoras especializadas é altamente dependente de oportunidades para a prática, encorajamento, ensino de qualidade e o contexto ecológico do ambiente" (GALLAHUE; OZMUM, 2005, p.368). Um ponto importante a ser destacado é que durante a manutenção das habilidades, a pontuação não caiu abruptamente, o que significa que o aprendizado não ocorreu só durante a intervenção, mas sim que se manteve estável durante o período da manutenção. Em alguns momentos, na manutenção, houve aumento da pontuação. Isso pode ser explicado pela repetição do movimento que resultou na aquisição da habilidade de rebater. Silvieira (2010) destaca a importância da repetição para a aprendizagem de uma habilidade motora. Para Manoel (1994) a repetição do movimento resulta em um padrão estável da habilidade motora em questão. 
A estabilidade das habilidades que permaneceram em linha de base durante a introdução do ensino de outra habilidade, apresentadas no gráfico, aponta que o aprendizado de uma habilidade não interfere na outra, ou seja, o ensino do forehand não influencia no ensino do backhand e assim sucessivamente. As manutenções das três habilidades permaneceram estáveis, para Cozby (2003) esse dado indica que o aprendizado de uma habilidade não é dependente da sessão de ensino, quando a sessão de ensino é retirada, a pontuação permanece estável, pois o aprendizado já foi interiorizado.

\section{CONCLUSÃO}

O delineamento do sujeito único é um método que permite observar a manipulação de variáveis, no presente estudo: o programa de ensino de badminton e a aquisição das habilidades manipulativas. O desenvolvimento uma habilidade motora pode ser analisada por meio desse delineamento pois permite observar a evolução do sujeito na aquisição do movimento, levando em consideração sua própria linha de base, e sem compara-la a outro sujeito. Isso implica em dizer que este método, considera as especificidades do sujeito sem generalizar o aprendizado.

Diante dos dados expostos acima, conclui-se que utilizar o delineamento do sujeito único, no caso, de linha de base múltipla foi eficaz para analisar a evolução do aprendizado do aluno na aquisição das habilidades motoras de rebater do badminton (forehand; backhand;saque). A utilização desse método auxilia na evolução de um programa de ensino uma vez que, permite ao professor verificar se a aprendizagem está sendo eficaz e se o aluno tem evoluído no treinamento

\section{REFERÊNCIAS}

APOLONI, Bruna Felix; LIMA, Flávia Evelin Bandeira; VIEIRA, José Luiz Lopes. Efetividade de um programa de intervenção com exercícios físicos em cama elástica no controle postural de crianças com. Revista Brasileira de Educação Física e Esporte, São Paulo, v. 27, n. 2, p.217-223, abr. 2013, https://doi.org/10.1590/S1807-55092013005000013.

COZBY, P.C. Métodos de pesquisa em ciências do comportamento. São Paulo. Editora Altas. 2003. $203 p$.

DALLA DÉA, V. H. S.; BALDIN, A. D.; DALLA DÉA, V. P. B. Informações gerais sobre a Síndrome de Down. In: DÉA DALLA, V. H. S. e DUARTE, E. Síndrome de Down: informações, caminhos e histórias de amor. São Paulo: Phorte, 2009, p.22-42

DUARTE, O. História dos Esportes. Senac .São Paulo, 2003 - 559 p.7

FLORES,M.I. BANKOFF,A.D.P. Influência da dança expressiva sobre o equilíbrio corporal em portadores com síndrome de Down. Revista da Faculdade de Educação Física da UNICAMP, Campinas, v. 8, n. 3, p. 35-46, set./dez. 2010

GALLAHUE, David L; OZMUN, John C. Compreendendo o desenvolvimento motor: bebês, crianças, adolescentes e adultos. São Paulo: Phorte, 2005. 585 p.

GAST, D. L. (2010). Single Subject Research Methodology in Behavioral Sciences. New York, NY: Routledge. $488 p$

Hallal CZ, Marques NR, Braccialli LMP. Aquisição de Habilidades Funcionais na Área de Mobilidade em Crianças Atendidas em um Programa de Estimulação Precoce. Rev Bras Crescimento Desenvolvimento Humano. 2008; 18(1): 27-34, https://doi.org/10.7322/jhgd.19863. 
LEITÃO, Maria Teresa Krahenbuhl. Procedimentos de ensino do tênis de campo para portadores de síndrome de Down. 1999. 97 f. Dissertação (Mestrado) - Programa de pós - graduação em Educação Física, Universidade de Campinas, Campinas, 1999.

LIMA, Ana Paula Rodrigues. Dança para síndrome de Down: uma análise dos benefícios da dança para portadores de síndrome de Down. Trabalho de Conclusão de Curso. Curso de Educação e Promoção da Saúde. Universidade de Brasília, 2010. p.55

MANOEL, Edison de Jesus. DESENVOLVIMENTO MOTOR: IMPLICAÇÕES PARA A EDUCAÇÃO FÍSICA ESCOLAR I.Revista Paulista de Educação Física, São Paulo, v. 8, n. 1, p.82-97, jan. 1994.

MATOS, M.A. Análise Funcional do Comportamento. Rev. Estudos de Psicologia, PUC-Campinas, v. 16, n.3, p. 8-18, setembro/dezembro 1999

OLIVEIRA, A.R.P. Programa de ensino das habilidades manipulativas do badminton em adolescentes com síndrome de Down. Dissertação de Mestrado. Programa de pós-graduação em educação especial, UFSCAR, 2016. 110p.

ORNELAS, M. A; SOUZA, C. A contribuição do profissional de educação física na estimulação essencial em crianças com síndrome de Down. Revista de Educação Física, Maringá, v. 12, n. 1, p.77-88, jan. 2001

PINTO, André Luis. O Ballet na vida de pessoas com síndrome de Down. Trabalho do de Conclusão de Curso. Departamento de Educação Física . Universidade de Brasília. 2013. p,53

SCHWARTZMAN, José Salomão. Síndrome de Down:São Paulo:Mackienzie,1999. 324 p.

SILVIEIRA, Sergio Roberto. Aquisição de habilidades motoras na educação física escolar: um estudo das dicas de aprendizagem como conteúdo de ensino. 2010. 149 f. Tese (Doutorado) - Curso de Pós Graduação em Educação Física, Usp, São Paulo, 2010.

SILVA, D R; FERREIRA, J S. INTERVENÇÕES NA EDUCAÇÃO FÍSICA EM CRIANÇAS COM SÍNDROME DE DOWN. Revista de Educação Física -Uem, Maringá, v. 2, n. 1, p.69-76, jan. 2001.

REIS FILHO, Adilson Domingos dos; SCHULLER, Juliana Aparecida de Paula. A CAPOEIRA COMO INSTRUMENTO PEDAGÓGICO NO APRIMORAMENTO DA COORDENAÇÃO MOTORA DE PESSOAS COM SÍNDROME DE DOWN. Pensar A Prática,Goiania, v. 13, n. 2, p.1-21, maio 2010.

WULF, Gabriele; TÖLLNE, Thomas; SHEA, Charles H. Attentional Focus Effects as a Function of Task Difficulty. Research Quarterly For Exercise And Sport, Eua, v. 78, n. 3, p.257-264, jan. 2007. Disponível em: http://www.psy.Imu.de/exp/people/ma/toellner/downloads/wulf-2007-rqes.pdf Acesso em: 25 de Setembro de 2017 